Revue d'histoire de l'Amérique française

REVUE D.HISTOIRE DE L'AMÉRIQUE FRANÇAISE

\title{
La ligue ouvrière catholique et l'organisation communautaire dans le Québec urbain des années 1940
}

\section{Jean-Pierre Collin}

Volume 47, numéro 2, automne 1993

URI : https://id.erudit.org/iderudit/305216ar

DOI : https://doi.org/10.7202/305216ar

Aller au sommaire du numéro

Éditeur(s)

Institut d'histoire de l'Amérique française

ISSN

0035-2357 (imprimé)

1492-1383 (numérique)

Découvrir la revue

Citer cet article

Collin, J.-P. (1993). La ligue ouvrière catholique et l'organisation

communautaire dans le Québec urbain des années 1940. Revue d'histoire de l'Amérique française, 47(2), 163-191. https://doi.org/10.7202/305216ar
Résumé de l'article

Durant les années 1940 et au début des années 1950, la Ligue ouvrière catholique (LOC) est un mouvement profondément partagé entre sa mission apostolique, qui est d'assurer le relèvement moral des familles ouvrières afin de contrer le spectre de la déchristianisation, et son engagement social, qui l'amène à vouloir contribuer à l'humanisation des conditions de vie et à freiner la prolétarisation de la classe ouvrière. Néanmoins, l'étude des modes d'organisation et des méthodes d'intervention, des réalisations et des stratégies d'engagement social de la Ligue (qui se dit à la fois mouvement ouvrier et mouvement familial) permet d'apprécier en quoi elle pose les jalons d'un modèle d'intervention et de développement de type communautaire. $\mathrm{Ce}$ modèle, dans son refus d'intégration à l'État providence, est facilement assimilé au conservatisme; il n'en constitue pas moins, nous semble-t-il, un héritage favorable à l'émergence, dans les années 1960, d'une animation sociale qui, bien que séculière et professionnelle, ne s'est jamais affranchie d'une certaine " déontologie catholique ».
Tous droits réservés @ Institut d'histoire de l'Amérique française, 1993
Ce document est protégé par la loi sur le droit d'auteur. L'utilisation des services d’Érudit (y compris la reproduction) est assujettie à sa politique d'utilisation que vous pouvez consulter en ligne.

https://apropos.erudit.org/fr/usagers/politique-dutilisation/ 


\title{
LA LIGUE OUVRIÈRE CATHOLIQUE ET L'ORGANISATION COMMUNAUTAIRE DANS LE QUÉBEC URBAIN DES ANNÉES 1940
}

\author{
JEAN-PIERRE COLLIN \\ INRS-Urbanisation
}

\section{RÉSUMÉ}

Durant les années 1940 et au début des années 1950, la Ligue ouvrière catholique (LOC) est un mouvement profondément partagé entre sa mission apostolique, qui est d'assurer le relèvement moral des familles ouvrières afin de contrer le spectre de la déchristianisation, et son engagement social, qui l'amène à vouloir contribuer à l'humanisation des conditions de vie et à freiner la prolétarisation de la classe ouvrière. Néanmoins, l'étude des modes d'organisation et des méthodes d'intervention, des réalisations et des stratégies d'engagement social de la Ligue (qui se dit à la fois mouvement ouvrier et mouvement familial) permet d'apprécier en quoi elle pose les jalons d'un modèle d'intervention et de développement de type communautaire. Ce modèle, dans son refus d'intégration à l'État providence, est facilement assimilé au conservatisme; il n'en constitue pas moins, nous semble-t-il, un héritage favorable à l'émergence, dans les années 1960, d'une animation sociale qui, bien que séculière et professionnelle, ne s'est jamais affranchie d'une certaine «déontologie catholique».

\section{ABSTRACT}

In the 1940's and early 1950's, the Ligue ouvrière catholique (LOC) was torn between its apostolic mission of bringing about the moral recovery of working-class families to counter the specter of religious indifference, and its commitment to social change which led it to act toward the humanization of living conditions and slow down the proletarianization of the working class. Nevertheless, through the study of the League's organization and intervention (which claimed to be both a labour movement and a family movement), one can appreciate how it pioneered a community-type model of intervention and development. Because it rejected integration into the welfare state, this model is often perceived as conservative; yet, it led to the emergence during the 1960's of a community organization movement that, while secular and professionally-run, retained aspects of a "Catholic ethic».

Ceux qui se sont intéressés à la «question urbaine» au Québec depuis la fin de la Deuxième Guerre mondiale ont salué l'apparition des comités de citoyens et des organismes populaires, au début des 
années 1960, comme un événement de toute première importance dans l'aménagement et la gestion de la ville.

Les sociologues et les politicologues ont surtout souligné la nouveauté d'un phénomène d'abord confiné aux quartiers du bas de la ville de Montréal, mais qui, dès la fin de la décennie, est observable dans la plupart des quartiers de Montréal, dans les banlieues anciennes et dans plusieurs centres urbains québécois de taille moyenne et grande. Le courant d'organisation communautaire en milieu urbain ou mouvement populaire des années 1960, argumente-t-on, représente, avec les syndicats et les partis politiques «ouvriers», une nouvelle composante du mouvement ouvrier.

Bien sûr, la difficulté d'enfermer les expérimentations dans des types ou des modèles ressort nettement des analyses faites jusqu'ici. Cependant, il appert que ces organisations se caractérisent d'abord par leur pragmatisme autant au niveau des objectifs que des processus organisationnels. On a observé que, au-delà de la diversité, de la variété des formes et des formules, elles s'affairent principalement à jeter les bases d'un réseau communautaire autonome ${ }^{1}$. Peu d'études toutefois ont cherché à le relier aux expériences d'animation sociale des décennies précédentes. Au contraire, on a beaucoup insisté sur la discontinuité marquée qui existerait entre ce «mouvement populaire» et le vide des décennies précédentes.

Sans nier la pertinence et l'utilité d'étudier les groupes surgis à cette époque et leur évolution depuis plus de trente ans, il nous apparaît également nécessaire de les retracer pour mieux comprendre les antécédents historiques. En effet, la diversité des interventions sur la scène locale au cours des années 1940 et 1950 est manifeste ainsi qu'on peut le voir, par exemple, dans l'analyse des modèles d'habitat expérimentés dans la région de $\mathrm{Montréal}^{2}$. Il appert, en particulier, que les milieux ouvriers et populaires ont manifesté un intérêt soutenu

1. Sur les comités de citoyens et les organismes populaires des années 1960 à 1975, voir, en particulier, Jacques Godbout et Jean-Pierre Collin, Les organismes populaires en milieu urbain: contre-pouvoir ou nouvelle pratique professionnelle? (Montréal, INRS-Urbanisation, «Rapport de recherche» $\mathrm{n}^{\circ} 3$, 1977); Pierre Hamel et Jean-François Léonard, Les organisations populaires, l'État et la démocratie (Montréal, Nouvelle Optique, 1981); Louis Favreau, Mouvement populaire et intervention communautaire de 1960 à nos jours: continuités et ruptures (Montréal, Centre de formation populaire/Les Éditions du Fleuve, 1989); Donald McGraw, Le développement des groupes populaires à Montréal (1963-1973) (Montréal, Les Éditions coopératives Albert Saint-Martin, 1979); Robert Boivin, Histoire de la Clinique des citoyens de SaintJacques (1968-1988) (Montréal, VLB éditeur, 1988).

2. Marc H. Choko, Jean-Pierre Collin et Annick Germain, «Le logement et les enjeux de la transformation de l'espace urbain: Montréal, 1940-1960», Urban History Review/Revue d'histoire urbaine, 15,2 (octobre 1986): 127-136 et 15,3 (février 1987): 243-253. 
pour la définition de ces modèles d'habitat et, surtout, qu'ils ont participé aux expérimentations en matière de logement et d'aménagement de l'espace urbain. Ces milieux n'ont pas fait que subir le développement urbain: ils ont parfois tenté d'en être les définisseurs ${ }^{3}$.

Sur un tout autre plan, notre attention ne peut manquer d'être attirée par l'interaction persistante entre les organismes populaires qui œuvrent en milieu urbain et les «catholiques sociaux», voire les organisations catholiques d'action sociale elles-mêmes. Les premiers comités de citoyens, le fait est connu ${ }^{4}$, sont le produit direct de l'Église, qui les a créés par l'intermédiaire des conseils des œuvres et de leurs animateurs (prêtres et laïcs). Pendant longtemps, bien que la plupart n'affichent ni caractère confessionnel ni orientation religieuse, les groupes populaires sont remarquablement nombreux à faire appel aux ressources financières et humaines de la paroisse et à prendre son territoire pour base d'opération. La sociabilité paroissiale a constamment fourni un environnement propice à l'implantation des organismes populaires. Ce lien privilégié est encore observable dans bien des cas. Plus significative encore est la présence soutenue des «actifs chrétiens ${ }^{5}$ », notamment dans les organisations jugées les plus innovatrices quant à leur domaine d'intervention ou à leur approche. Sans être complètement passée sous silence, cette interaction intime avec les catholiques sociaux n'a généralement pas suscité l'intérêt des analystes du «mouvement populaire».

Les deux expériences présentent, en effet, plusieurs similitudes rattachées pour une bonne part au fait que le centre d'intérêt de l'action soit, pour l'une et l'autre, l'amélioration des conditions de vie de la majorité des citadins. Sans vouloir forcer l'analogie ni «gommer» les différences, il nous a donc semblé pertinent de rapprocher l'expérience de la LOC des années 1940 de celle des comités de citoyens et des organismes populaires des années 1960 et 1970. Elles ont aussi un trait commun fondamental: la volonté de faire surgir dans la société civile un cadre permanent d'intervention qui, dans la recherche de solutions innovatrices, se substitue à l'État. L'objectif ultime est de favoriser l'éclosion d'une démocratie sociale qui dépasse les limites de la démocratie politique.

3. Jean-Pierre Collin, La cité coopérative canadienne-française: Saint-Léonard-de-PortMaurice, 1955-1963 (Montréal et Québec, INRS-Urbanisation et Presses de l'Université du Québec, 1986).

4. Voir Michel Corbeil, «Historique de l'animation sociale au Québec», Relations, 349 (mai 1970): 139-147; Jacques Godbout et Jean-Pierre Collin, op. cit., chapitre premier.

5. Raymond Courcy, Le rôle social et politique de l'Église catholique dans les quartiers populaires au Québec, thèse de doctorat, Université de Bordeaux I, 1981. 
L'examen de cette dimension particulière de l'histoire de la Ligue ouvrière catholique vient confirmer le rôle déterminant et incomparable de l'Action catholique spécialisée dans la diversification des stratégies d'action de l'Église catholique et dans le renouvellement de la pensée catholique au Québec ${ }^{6}$. Mais surtout, il en complète la lecture, car c'est le débordement social de la Jeunesse étudiante catholique (JEC) qui a été le plus largement mis en vedette par l'historiographie ${ }^{7}$. En particulier, l'arrivée de Cité libre, dont les fondateurs et les collaborateurs, dans les années 1950, étaient presque tous d'anciens dirigeants nationaux de la centrale étudiante, est vue comme un événement marquant de l'histoire du Québec. Ainsi, Michael Behiels accrédite-t-il la prétention des jécistes d'avoir été, à travers cette revue, à l'origine de l'émergence au Québec d'un certain libéralisme universalisant à la recherche d'une démocratie industrielle chrétienne ${ }^{8}$. Plusieurs d'entre eux ont aussi laissé leur marque dans le monde du journalisme québécois.

L'expérience de l'Action catholique ouvrière et surtout celle de la Ligue ouvrière catholique n'ont pas reçu la même attention. Pourtant, comme le suggère Louisette Pothier, il faut reconnaître dans l'Action catholique ouvrière une «force de mouvement» au même titre que le mouvement syndical et le mouvement coopératif 9 .

Dans les années 1940, «l'urbanisation ouvre à la pastorale de nouveaux champs d'action ${ }^{10}{ }_{\gg}$ qui, d'après Jean Hamelin, sont tout particulièrement pris en charge par les organisations d'Action catholique ouvrière. Pour certains, ces orientations nouvelles n'auront été qu'un compromis conjoncturel, qu'une tentative plus ou moins sophistiquée de récupération pour réactiver, si possible dans son intégrité, la doctrine sociale de l'Église ${ }^{11}$. D'autres, dont Charles Côté et Yannick

6. Là-dessus, voir, en particulier, Gabriel Clément, Histoire de l'Action catholique au Canada français (Montréal, Fides, 1972) et «L'Action catholique: les mouvements spécialisés à Montréal de 1930 à 1966», Rolland Litalien, dir., L'Église de Montréal, 1836-1986: aperçus d'hier et d'aujourd'hui (Montréal, Fides, 1986); Jean Hamelin et Nicole Gagnon, Histoire du catholicisme québécois: le XXe siècle (Montréal, Boréal Express, 1984), 2 tomes.

7. Parmi les organisations d'Action catholique spécialisée, la JEC est la seule à avoir fait l'ojet de quelques mémoires de maîtrise. C'est aussi la seule à avoir produit sa propre histoire. Voir Jeunesse étudiante catholique, La J.É.C., école de responsabilité au cœur du milieu étudiant: jalons pour une histoire de la J.É.C., 1953-1985 (Montréal, 1985).

8. Michael Behiels, Prelude to Quebec's Quiet Revolution. Liberalism Versus NeoNationalism, 1945-1960 (Montréal et Kingston, McGill-Queen's University Press, 1985).

9 Louisette Pothier, «L'Action catholique ouvrière: un aspect des rapports entre l'Église et les travailleurs», Jean Hamelin et Fernand Harvey, dir., Les travailleurs québécois, 1941-1971 (Québec, Université Laval, Institut supérieur des sciences humaines, «Instrument de travail» no 20 , août $1976,513$.

10. Jean Hamelin, Histoire du catholicisme québécois: le XXe siècle (Montréal, Boréal Express, 1984), II: 68.

11. À titre d'exemple, voir Yves Vaillancourt, L'évolution des politiques sociales au Québec, 1940-1960 (Montréal, Les Presses de l'Université de Montréal, 1988). 
G. Harnois, en font une lecture moins univoque et mettent l'accent sur son caractère ambivalent de mouvement partagé entre ses origines doctrinales et sa pratique sociale. Tout en admettant que cette avantgarde devait permettre à l'institution ecclésiale de s'adapter éventuellement aux réalités nouvelles, l'Action catholique spécialisée est alors présentée comme la "première expérience structurée d'animation au Québec ${ }^{12}$ ». On doit néanmoins s'étonner de ce que leur analyse, centrée sur la JOC et la JEC, ignore presque la Ligue ouvrière catholique canadienne (LOC), une organisation qui, comme on le verra, a fourni sa part de militants à l'animation sociale.

\section{VOIR - JUGER - AGIR}

Mouvement d'action catholique spécialisée mis en place entre 1938 et 1942, la LOC a survécu jusqu'au milieu des années 1960. À l'instar de son pendant français ${ }^{13}$, dont elle s'inspire étroitement au cours des premières années, la Ligue a été fondée pour relever un double défi. D'une part, au nom de la doctrine sociale catholique, elle voulait assurer le relèvement moral des familles ouvrières et contrer ainsi le spectre de la déchristianisation. D'autre part, elle souhaitait contribuer à l'humanisation des conditions de vie en ville et freiner la prolétarisation de la classe ouvrière.

Pour mener cette œuvre de restauration morale et sociale, elle entend s'imposer, sous la double étiquette de mouvement ouvrier et de mouvement familial, comme la représentante de la masse ouvrière qui, pour elle, comprend l'ensemble des cols blancs salariés et des cols bleus, et tous ceux qui sont victimes à des degrés divers d'insécurité et de dépersonnalisation dans leur vie active, sociale et quotidienne. La LOC est donc intervenue largement dans le champ des réponses publiques et privées qui ont été organisées pour résoudre les problèmes liés aux conditions de vie en milieu urbain, au sortir de la Deuxième Guerre mondiale, notamment le problème de l'habitation.

L'analyse de l'insertion de la LOC dans la société civile en faveur «d'un monde ouvrier plus humain ${ }^{14} »$ fait ressortir d'emblée la

12. Charles Côté et Yannick G. Harnois, Le développement de l'animation au Québec: sources, apports et limites, mémoire de maîtrise (sociologie), Université du Québec à Montréal, 1977.

13. Sur la Ligue ouvrière catholique française, voir Joseph Debes, Naissance de l'Action catholique ouvrière (Paris, Les Éditions ouvrières, 1982); les Cahiers du Groupement pour la recherche sur les mouvements familiaux (Forest-sur-Marque); Bruno Duriez, «D'un mouvement totalisant à des groupements d'action catholique spécialisée. L'exemple d'associations françaises d'origine confessionnelle», Roger Levasseur, dir., De la sociabilité. Spécificité et mutations (Montréal, Boréal, 1990), 183-199.

14. Slogan inscrit en sous-titre du Front ouvrier, l'hebdomadaire de la Ligue, le premier mois de sa parution (décembre 1944). 
difficulté qu'elle éprouve à harmoniser durablement ses rôles de mouvement apostolique et de mouvement social. D'ailleurs, au cours des années 1950 et 1960 , sa force d'attraction et la portée de ses interventions sont de plus en plus réduites et elle délaisse le champ de l'action sociale pour se replier derrière sa mission apostolique. Mais, entre 1942 et les années 1952-1954, la LOC nationale a d'abord interprété sa «mission» en insistant sur l'urgence de réformer les rapports sociaux ${ }^{15}$.

Ainsi, dans une volonté clairement et souvent exprimée de se distinguer des bonnes œuvres, la LOC insiste sur l'importance de sa mission sociale, la transformation et l'humanisation du milieu de vie (ouvrier). L'expansion et la notoriété de la Ligue sont le résultat direct de cet apostolat social au sein de la masse ouvrière. Plus particulièrement dans les années 1940, les effectifs et le rayonnement de la LOC augmentent de façon soutenue dans les principaux centres urbains du triangle Montréal-Sherbrooke-Québec ${ }^{16}$. Parallèlement, l'hebdomadaire Le Front ouvrier fait particulièrement bonne figure dans l'univers de la presse engagée et se vend aussi bien en région qu'à Montréal.

Pour bâtir la cité nouvelle, la LOC ambitionne avant tout d'être l'université de la classe ouvrière, une école de vie chrétienne familiale et sociale dont la pédagogie repose sur la méthode d'enquête inspirée par le mot d'ordre de Cardjin: Voir - Juger - Agir. Par opposition au reportage ou à la recherche pure, cette méthode de «formation par l'action et dans l'action» serait, prétend-on, «adaptée à la grande

15. L'analyse qui suit reprend le chapitre 5 de Jean-Pierre Collin, La Ligue ouvrière catholique canadienne, 1937-1965: un mouvement social urbain, thèse de doctorat (histoire), Université du Québec à Montréal, décembre 1990. Cette étude repose principalement sur les archives administratives de la LOC nationale et sur ses publications. Les archives administratives, conservées pour la plupart aux Archives nationales du Québec à Montréal (Fonds de la LOC-MTC; Dossier numéro 06,M-P257), comprennent notamment la correspondance, les documents préparatoires ainsi que les rapports de chaque réunion des instances nationales de la Ligue, divers procès-verbaux, des documents de fonds et les bulletins mensuels publiés à l'intention de diverses catégories de militants à l'intérieur de la LOC. Dans le cas des publications, devant la masse de documentation, un choix s'imposait. Ne faisant du magazine annuel qu'une lecture en diagonale, nous avons concentré notre attention sur les journaux $-L e$ Mouvement ouvrier, mensuel publié d'avril 1939 à novembre 1944 et Le Front ouvrier, hebdomadaire qui a pris la relève du premier et qui est paru sans interruption jusqu'en mars 1954.

16. Au début des années 1950 , la Ligue peut compter sur 3800 militantes et sur près de 1900 militants. La présence majoritaire des femmes est caractéristique des asssociations volontaires à vocation sociale en général et des œuvres catholiques en particulier; la proportion d'hommes est comparativement plus élevée à la LOC que dans les autres mouvements d'action catholique spécialisée. Sans appartenir aux catégories de revenu les plus faibles, les membres de la LOC proviennent pour la plupart «de la classe ouvrière et employée» (les femmes sont ménagères, épouses d'ouvriers). 
majorité des gens qui ne sont pas des intellectuels ${ }^{17}$ ». Elle est fondée sur une démarche inductive qui part du vécu des militants et dont le produit recherché n'est pas une analyse savante, mais un instrument pour l'action. Sans négliger pour autant la contribution de la LOC à l'épanouissement intellectuel et social des ouvriers, à l'émergence et au développement d'une culture ouvrière originale, cette démarche est, affirme-t-on, «conçu(e) pour aboutir à une action organisée par le mouvement ${ }^{18}$ ».

Appelé à devenir «un enquêteur ambulant qui observe, juge et réagit à cœur de jour: chez lui, dans le tramway, à l'usine, au cinéma, aux réunions de syndicats, dans les familles qu'il visite, etc. ${ }^{19}{ }^{\prime}$, le militant lociste est engagé, pour un an, dans une démarche collective, planifiée à l'avance, sur une tranche particulière de la vie ouvrière. Cette enquête programme, qui est au cœur de la réunion bihebdomadaire du cercle d'étude, est parfois jumelée à une grande enquête statistique. La Ligue met alors à profit son réseau de militants pour recueillir une masse de données (sur le coût de la vie en 1943, sur l'habitation en 1947, sur le problème des relations entre la famille et l'école en 1951 et sur les loisirs en 1953). Encore ici, ce n'est pas la valeur proprement scientifique de l'enquête (le respect des règles de la statistique) qui importe, mais la valeur heuristique d'une démarche tournée vers l'action.

\section{UN DOUBLE APOSTOLAT: LA FAMILLE ET LE MILIEU SOCIAL}

Les premières années, l'enquête sociale sert strictement à faire prendre conscience de l'étendue des problèmes de la famille ouvrière et des nombreuses facettes de la situation de celle-ci. Vraisemblablement sensibles au contenu de la récente lettre pastorale collective des évêques de la province de Québec sur la restauration sociale ${ }^{20}$,

17. La technique de l'enquête sociale est définie par bribes dans les bulletins internes, dans les brochures ainsi que dans les programmes et les rapports des sessions intensives. Le «manuel de la LOC» (Les comités nationaux de la LOC, La Ligue ouvrière catholique, mystique et technique (Montréal, Fides, 1947)) y consacre quelques pages. Ce n'est, toutefois, qu'au début de 1954 qu'est finalement produit un document de synthèse, «La méthode d'enquête», document d'orientation remis aux participants au Conseil national de la LOC des 13-14 mars 1954, 24 pages. Repris dans Léopold Séguin, «La méthode d'enquête», L'Action catholique ouvrière, 4,4 (avril 1954): 138-159.

18. Rapport du Conseil national de la LOC des 14-15 novembre 1964, 15.

19. «La méthode d'enquête», document d'orientation remis aux participants au Conseil national de la LOC des 13-14 mars 1954, 11.

20. La restauration sociale, Lettre pastorale collective de S. Ém. le Cardinal Archevêque de Québec et de Leurs Excellences les Archevêques et Évêques de la province de Québec à l'occasion de l'anniversaire des encycliques «Rerum novarum» et «Quadragesimo anno», 11 mars 1941 (Montréal, École Sociale Populaire, 1943). 
Figure 1

Thèmes des enquêtes sociales et familiales de la LOC entre 1939 et 1962

\begin{tabular}{lll}
\hline Année & \multicolumn{1}{c}{ Enquête sociale } & \multicolumn{1}{c}{ Enquête familiale } \\
\hline $1939-40$ & La famille ouvrière & \\
$1940-41$ & $\begin{array}{l}\text { La situation actuelle de la } \\
\text { classe ouvrière }\end{array}$ & \\
$1941-42$ & Le mariage et la société & \\
$1942-43$ & L'éducation des enfants de 0 à 7 ans & Le mariage \\
$1943-44$ & L'habitation ouvrière & Le travail \\
$1944-45$ & Les coopératives d'habitation & L'éducation des enfants de 7 à 14 ans \\
$1945-46$ & Les coopératives de crédit et de & Le mariage, vocation apostolique \\
& consommation & L'éducation des enfants de 14 à 20 ans \\
$1946-47$ & Le revenu familial & Le devoir familial \\
$1947-48$ & La santé de la famille ouvrière & \\
$1948-49$ & Le coût de la vie & \\
$1949-50$ & Le sens social chrétien & \\
$1950-51$ & Famille-École & \\
$1951-52$ & Pères et mères de famille & \\
$1952-53$ & Les loisirs de la famille ouvrière & \\
$1953-54$ & À la conquête de la vraie sécurité & \\
& ouvrière & \\
$1954-55$ & Vie conjugale \\
$1955-56$ & Le travail \\
$1956-57$ & Le civisme \\
$1957-58$ & Le sens communautaire de l'argent & \\
$1958-59$ & Le corps, moyen de se spiritualiser & \\
$1959-60$ & ou de s'animaliser & Soyons fiers, ouvriers \\
$1960-61$ & Pour une vie de travail plus humaine & \\
$1961-62$ & A la découverte de l'amour véritable & \\
\hline
\end{tabular}

les dirigeants nationaux proposent un virage en septembre $1941^{21}$. Ils font valoir la nécessité de déboucher sur une «action sociale concertée ${ }^{22}$ », sur une implication accrue de la LOC dans la communauté.

En 1942-1943, l'enquête délaisse d'ailleurs les grandes fresques pour se concentrer sur des aspects particuliers, spécifiques de la vie ouvrière, d'une part, de la vie familiale, d'autre part. Plus qu'un lieu de socialisation où l'individu apprend à connaître et à remplir ses devoirs, avant de réclamer ses droits, la famille est alors vue comme un élément d'une société en changement. Elle (la famille) devient le point d'appui d'une stratégie d'intervention dans les problèmes collectifs du milieu ouvrier. C'est pour faire place à cette double lecture qu'entre 1942 et 1949 l'enquête sociale est séparée de l'enquête familiale, et que la LOC nationale multiplie les interventions sur la place publique.

S'ouvre alors un cycle d'enquêtes plus sociales que familiales qui confirme le virage social annoncé par la LOC elle-même (figure 1).

21. L'année lociste se déroule de septembre à août.

22. Rapport du premier Conseil national de la LOC, les 5-6 septembre 1941. 
Sont d'abord étudiés, pendant deux années consécutives, les problèmes du logement et les solutions qui peuvent être apportées, principalement le «crédit urbain ${ }^{23}$ » et les coopératives d'habitation. Le problème du niveau de vie et du pouvoir d'achat vient en second lieu. Pour améliorer son sort, plusieurs stratégies sont suggérées à la classe ouvrière, telles que la mise sur pied de coopératives et l'organisation de ligues de consommateurs. Une année d'étude est consacrée à la santé des familles ouvrières, en mettant l'accent sur les causes environnementales des problèmes: logements insalubres ou exigus, insécurité du revenu, alimentation déficiente, loisirs inappropriés.

Pour faire «sortir» l'enquête sociale de la section paroissiale et de son aire d'influence immédiate, la LOC fait appel à une variété d'instruments de propagande: tracts, assemblées populaires et forums, mais surtout magazine annuel et journal hebdomadaire notamment dans sa partie éditoriale. Au sortir de la guerre, la direction nationale sent le besoin d'élargir la portée de son action d'éducation populaire. Elle se donne une tribune de premier ordre avec la semaine annuelle de la famille ouvrière ${ }^{24}$.

Ce sont les enquêtes sur les conditions de vie de la classe ouvrière, à commencer par celle sur le logement, qui fournissent la matière aux premières semaines de la famille ouvrière. Ces enquêtes ont d'autant plus de prestige qu'elles servent, à cette époque, de conclusion à des séries de forums et d'assemblées populaires portant sur les mêmes thèmes: le logement en 1947, la santé en 1948 et le budget familial en 1949.

Toutes les techniques de propagande sont alors mises en œuvre de façon coordonnée: numéro spécial du journal, tracts (brochures), forums, jeux scéniques, chants, etc. La semaine de la famille ouvrière est aussi une grande opération médiatique où l'on tente de mettre à profit la grande presse et la radio.

Sur le plan de la propagande et de l'éducation populaire, l'action de la LOC est ainsi dominée par son implication dans les grands problèmes de l'après-guerre en matière de conditions de vie du monde

23. Le crédit urbain ou crédit ouvrier à l'habitation est une proposition spécifique d'aide gouvernementale à l'accession à la propriété individuelle qui fut revendiquée par de nombreux groupes sociaux de la fin des années 1930 au milieu des années 1950, notamment la CTCC, le Conseil supérieur de la coopération et la LOC. Il est ainsi désigné par analogie avec le crédit agricole.

24. Le lecteur intéressé trouvera une analyse de ces semaines de la famille ouvrière en relation avec l'évolution des thèmes de l'enquête sociale dans Jean-Pierre Collin, op. cit., 185203. 
ouvrier. Cela alimente et va de pair avec une approche originale de l'action sociale catholique.

\section{L'ENTR'AIDE FAMILIALE OUVRIÈRE}

«La LOC, affirme-t-on, ne doit pas rester dans le domaine du spirituel $\operatorname{pur}^{25}$.» Son projet éducatif n'a de chances de réussir qu'arrimé aux besoins concrets de ceux qu'elle veut atteindre et mobiliser. C'est pourquoi, chaque enquête sociale, entre 1943 et 1949, se termine par un appel à l'engagement temporel.

C'est dans cet esprit qu'est lancée officiellement l'Entr'aide familiale ouvrière ${ }^{26}(E F O)$, un ambitieux programme d'intervention sociale et économique. La LOC se décrète maître d'œuvre et centre nerveux d'un réseau d'entr'aide aux ramifications des plus étendues, comme on s'en rendra compte à la lecture de l'organigramme théorique de l'EFO, reproduit à la figure 2 . Ses activités se déploieront dans chaque paroisse, ville et région où elle est présente aussi bien qu'à l'échelle nationale. L'action de la LOC ne s'adresse pas seulement aux consciences; elle doit soutenir les initiatives qui ont pour but d'assurer concrètement la transformation des conditions de vie des familles ouvrières.

À l'origine, la structure de l'Entr'aide familiale ouvrière est déterminée par la recherche d'un mode d'intervention basé sur la cohabitation de deux stratégies complémentaires: assurer la plus grande efficacité des ressources existantes et provoquer la création de nouveaux services lorsque ces ressources s'avèrent inexistantes ou simplement insuffisantes.

Dans le premier cas, la LOC cherche à développer une expertise d'agent de liaison (ou de centre de référence) entre les besoins des familles ouvrières et les services publics, les œuvres économicosociales, les associations volontaires, etc. À ce stade, il s'agit moins de prendre l'initiative que de mettre en évidence ou de relancer ce qui existe déjà. Surtout, pour maximiser la portée de leurs interventions ou pour en augmenter l'envergure, les locistes, prévoit-on, devront chercher activement à se tailler une place d'auxiliaires bénévoles auprès des techniciens du service social, de la santé ou des loisirs.

C'est avec beaucoup d'ambition et de ferveur que la Ligue aborde son rôle d'intermédiaire. Son projet d'ailleurs n'est pas modeste. Tout

25. Meneurs (bulletin mensuel interne de la LOC), 3,9 (juin 1943): 1. Repris dans Pour la conquête des foyers ouvriers (bulletin mensuel interne de la LOC), 6,2 (novembre 1944): 2.

26. «Pour des foyers nouveaux dans une cité nouvelle», programme de la première session intensive, Montréal, les 22-23 août 1942 et «L.O.C. coopérative de bonheur», programme de la deuxième session intensive, Québec, les 31 juillet et $1^{\text {er }}$ août 1943. 
en se plaçant à la remorque d'initiatives prises par d'autres, elle ne se voit pas comme un collaborateur occasionnel au rôle effacé, mais cherche à s'imposer en tant que coordonnateur général. Elle veut exercer ce rôle aussi bien à l'échelle de la ville ou de la région, par ses fédérations diocésaines, qu'à celle de la paroisse dont elle est une des institutions officielles (figure 2).

Cette approche domine nettement les débuts de l'Entr'aide familiale ouvrière. Elle a donné en certains endroits des résultats appréciables avec la prolifération des "petits services», particulièrement nombreux dans les domaines du dépannage économique, de l'économie domestique, de la médecine familiale et des loisirs. Les structures de ces petits services sont des plus rudimentaires. Ils ne touchent pas plus d'une ou deux paroisses. C'est toutefois la seconde stratégie qui devient bientôt dominante et plus significative.

Les sections locistes ont tôt fait l'inventaire des œuvres et des ressources locales et mis en place les relais nécessaires à leur bon fonctionnement. Après avoir annoncé que «c'est maintenant le temps d'organiser des services bien à nous ${ }^{27}{ }_{\nu}$, les militants se lancent donc dans la fondation et dans l'organisation d'un ensemble de services nouveaux qui doivent faire de la LOC une «coopérative de bonheur ${ }^{28}$ ». L'Entr'aide familiale ouvrière est, de plus en plus, dominée par ce désir de prendre en charge «toutes les misères, toutes les difficultés, toutes les souffrances de la classe ouvrière ${ }^{29} \gg$.

On assiste à une multiplication des initiatives locales les plus variées (figure 3): camps et autres loisirs familiaux, œuvres des terrains de jeu, cours d'arts ménagers qui mènent éventuellement à la fondation d'instituts, services péri et post nataux en liaison étroite avec les cliniques, jardins ouvriers auxquels sont greffées des expériences coopératives sectorielles (coopérative de pommes de terre à Drummondville, coopérative de mise en conserve à Beauharnois), écoles de formation (ou collèges ouvriers) à Montréal et à SaintHyacinthe. On insiste toutefois sur le caractère éphémère de l'implication de la LOC dans la création de «services sociaux»: «dès que l'entreprise dépasse ses cadres, écrit-on, il lui faut en confier la direction à d'autres. Ces autres-là pourront être des membres de la LOC; ils ne seront pas la LOC $^{30}$.»

27. Pour la conquête des foyers ouvriers (bulletin mensuel interne de la LOC), 4,5 (février 1943): 2.

28. Lancé à la session intensive de 1943, «La LOC, une coopérative de bonheur» est un slogan abondamment utilisé, jusqu'à la fin des années 1940, dans les bulletins mensuels et dans Le Front ouvrier.

29. Ligue ouvrière catholique, LOC! Espoir des familles ouvrières (Montréal, Les Éditions de la L.O.C., 1947), 49.

30. Meneurs (bulletin mensuel interne de la LOC), 4,5 (février 1944): 2. 
Figure 2

Organigramme de l'Entr'aide familiale ouvrière annexé au programme de la session intensive d'août 1942

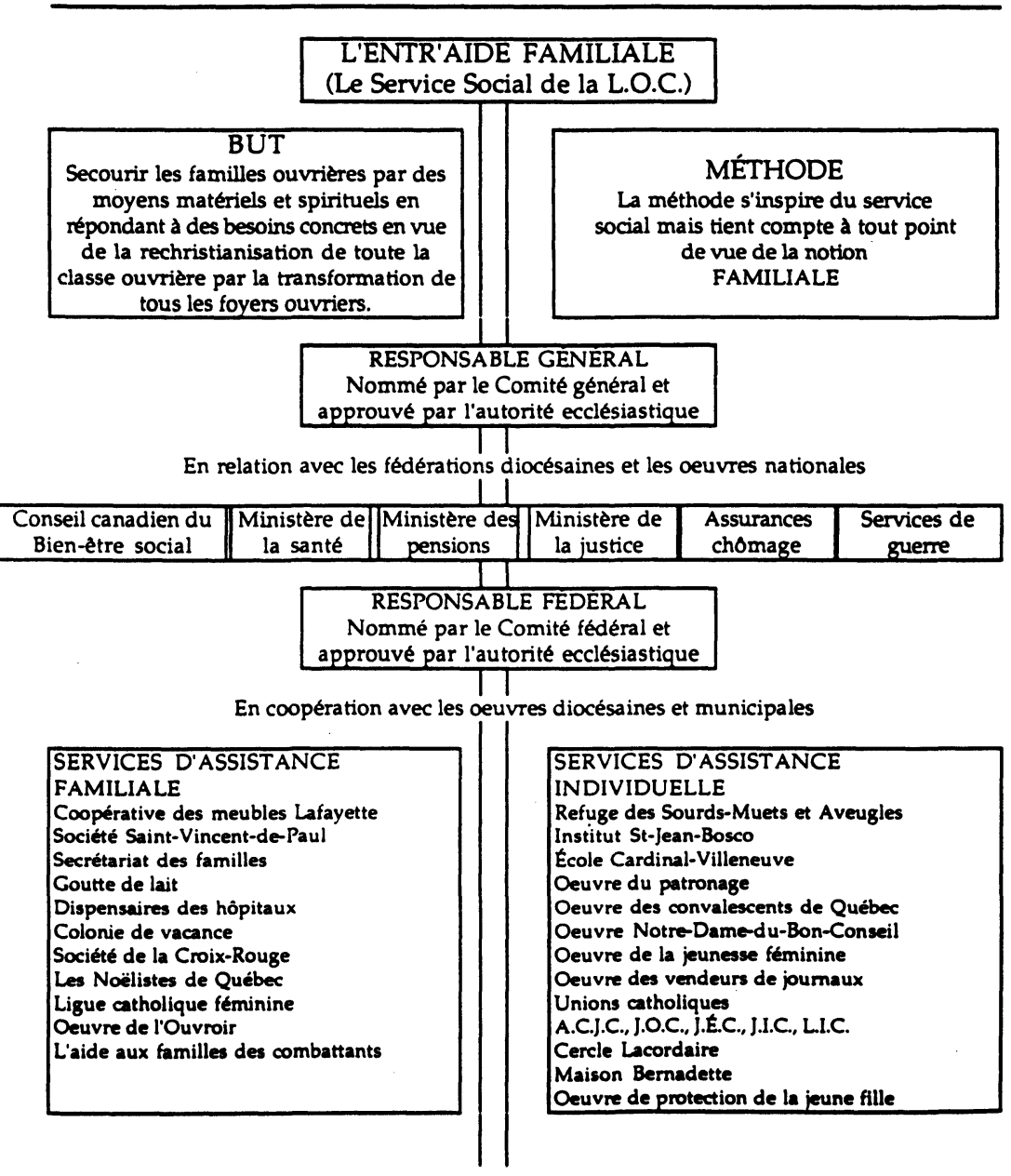




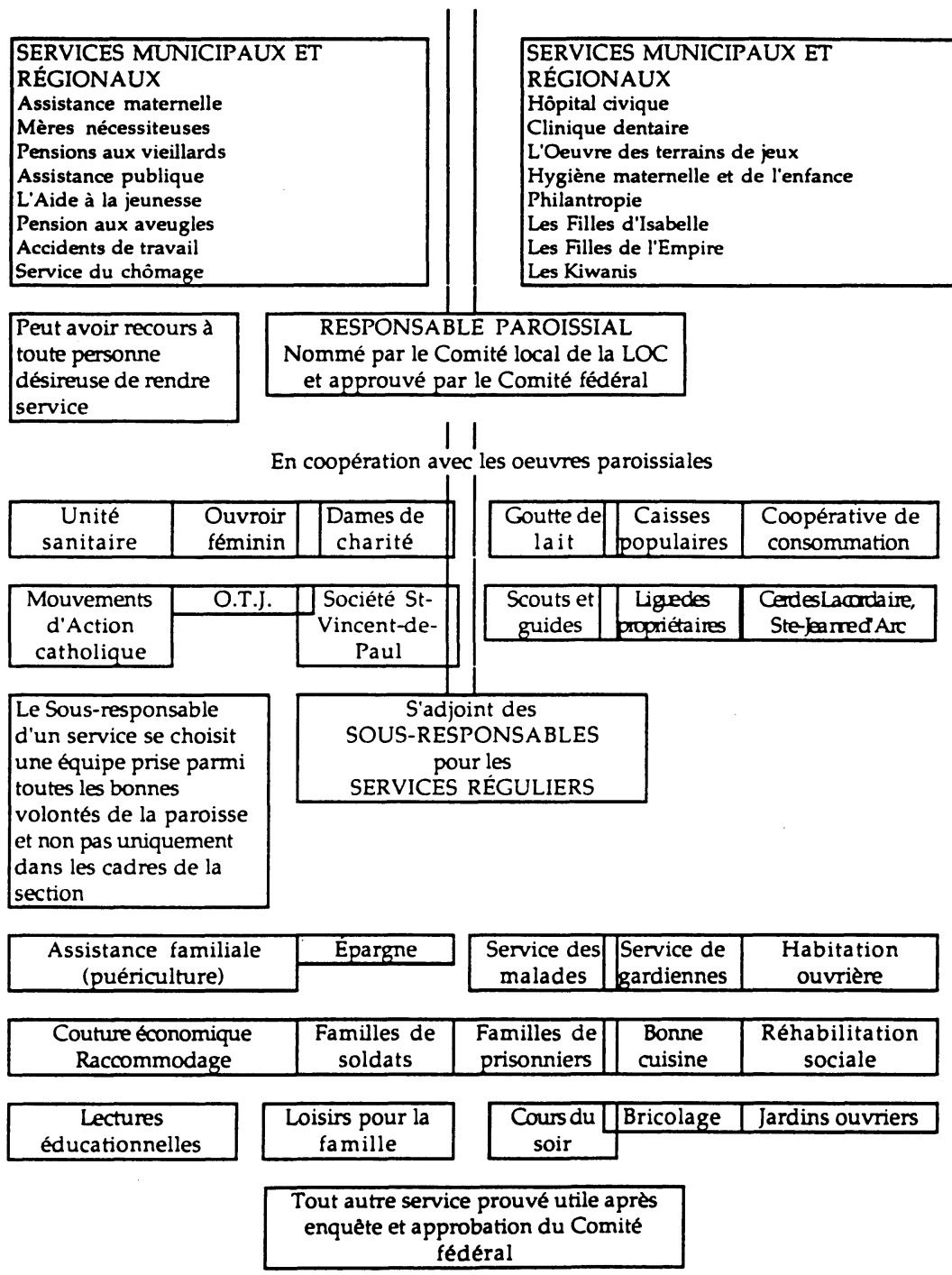

N.B. Pour toute question locale insoluble par le Responsable Paroissial on doit référer au Responsable Fédéral 
Figure 3

Les petits services à Drummondville et à Trois-Rivières/Shawinigan/ Cap-de-la-Madeleine, au milieu des années 1940

\begin{tabular}{|l|l|}
\hline \multicolumn{1}{|c|}{ Drummondville } & \multicolumn{1}{c|}{ Trois-Rivières } \\
\hline Camps familiaux & Villas ouvrières \\
Loisirs cinéma & Bibliothèque \\
Centre de loisirs (projet) & Service d'anniversaires \\
Soirées familiales & Service des vacances des mamans \\
Jardins ouvriers & \\
Coop. de pommes de terre & \\
Coop. de tomates & \\
Service de gardiennes & \\
Asssistance aux familles & Service de gardiennes \\
Aide maternelle & Aide aux mamans malades \\
Éducation sociale & Assistance maternelle \\
Budget familial & Cours d'économie domestique \\
& Budget familial \\
Vestiaire & Visites aux malades \\
& Transport vers l'unité sanitaire \\
& Comptoir d'échange \\
& Assistance à la confection de \\
Déménagements & vêtements \\
Paniers de Noël & Placement d'aides familiales \\
Rapports d'impôt & Déménagements \\
Service d'imprimerie & Paniers de Noël \\
\hline
\end{tabular}

Source : "En avant Nicolet...», Le Mouvement ouvrier, vol. V, no 10-11, p. 15, et Léo Girard, ptre, «Panorama des services locistes de la Mauricie», L'Action catholique ouvrière, vol. III, no 7, juillet-août 1953, p. 348-360 (inventaire de ce qui existait vers 1947).

Néanmoins, les expériences locales sont rapidement placées dans l'ombre des tentatives de bâtir les «grands services» nationaux de la LOC. Ces derniers exigent du mouvement une participation prolongée et font partiellement oublier que ce sont les militants individuellement, et non la LOC collectivement, qui doivent s'engager sur le plan social.

\section{LES GRANDS SERVICES DES ANNÉES 1940}

Dans les années 1940, quatre domaines d'activité ont ainsi été coordonnés par les instances diocésaines ou organisés directement à l'échelle nationale et ont contribué à générer «une nouvelle façon de présenter la L.O.C. ${ }^{31}{ }^{»}$ : les camps familiaux, les jardins ouvriers, le

31. Rapport de la réunion du 3 décembre 1942 du comité national de la LOC, 2. 
service du budget familial ${ }^{32}$ et le service de l'habitation ouvrière, de loin le plus important.

Pour faire contrepoids aux loisirs commercialisés, que l'on juge source de déchéance morale, les locistes organisent des camps familiaux. À la fin des années 1940, pratiquement toutes les fédérations locistes gèrent une ou plusieurs "coopératives de grand air et de repos $^{33}$ ». Ces modestes «cités ouvrières de vacances», comme on aime les appeler, comprennent, généralement, en bordure d'un plan d'eau, un complexe de chalets d'été, offerts en location pour des périodes d'une ou deux semaines, auquel s'ajoutent souvent un pavillon communautaire, une plage, des espaces pour pique-niquer ou le camping, etc.

Le service des jardins ouvriers prend racine, en 1941 également, à Drummondville, Québec et Montréal. Dans le dernier cas, la LOC collabore étroitement avec la Community Garden League of Greater Montreal, un organisme qui avait déjà quelques années d'expérience en la matière. La LOC nationale accorde une grande importance aux jardins ouvriers qu'elle considère comme un service polyvalent offrant des avantages économiques et sociaux variés. Ainsi, dans les nombreux articles que leur consacre le journal, on en fait, tour à tour: a) une œuvre de restauration morale - conserver à la famille ouvrière ses racines champêtres, garder les pères à la maison; b) une initiation aux bienfaits de la propriété; c) une source de loisirs sains, au grand air et en famille; d) un bon coup de pouce au budget familial; e) une façon d'acquérir une meilleure alimentation.

Les autres fédérations ayant emboîté le pas, le service des jardins ouvriers est, entre 1943 et 1947, le plus important par le nombre des familles mobilisées. À l'échelle de la province, les bulletins internes font régulièrement mention de quelque 2000 à 2500 jardins potagers, le plus souvent aménagés sur des lots appartenant aux municipalités. En 1946, à Montréal seulement, il y en aurait eu près d'un millier. Après la guerre, la LOC souhaite assurer la permanence de la collaboration financière des municipalités. Elle tente de les convaincre d'insérer dans les plans d'urbanisme, au même titre qu'elles le font pour les parcs, des emplacements réservés aux jardins ouvriers. Cette

32. Deux autres services, inspirés de la formule du Service de préparation au mariage de la JOC, sont aussi fondés dans les années 1940: le Service d'orientation des foyers (SOF) et les Cercles sociaux féminins (CSF). Leur popularité n'est toutefois assurée que dans les années 1950, après que la LOC se soit déclarée plus soucieuse d'influencer les relations humaines à l'intérieur de la famille ouvrière que de s'attaquer à ses besoins temporels immédiats. Le premier service s'adresse au couple dont il veut animer la vie conjugale. Réservés aux femmes célibataires, les CSF projettent d'animer leur vie sociale et philanthropique.

33. Le Front ouvrier, 4,41 (4 septembre 1948): page titre. 
proposition de municipalisation mitigée ${ }^{34}$ n'a vraisemblablement pas eu de suites. À partir de 1948, la LOC se désengage progressivement de l'organisation des jardins ouvriers qui, par ailleurs, commencent à perdre de leur popularité. À la place, elle mène pendant quelques années une campagne d'éducation et de formation afin de propager la pratique du «jardin familial».

Dès 1942, pour des considérations morales autant qu'économiques, encore une fois, la LOC s'intéresse à la technique du budget familial. Elle élabore donc progressivement un «cahier du budget» dont la version finale est publiée en 1949 et rééditée chaque année jusqu'en $1964^{35}$. La vente du cahier du budget atteint 25000 exemplaires en 1949, l'année de l'enquête sociale sur le coût de la vie et de la Semaine de la famille ouvrière sur le budget familial. Entre 1944 et 1948, il s'en était vendu 7000 par an, en moyenne. Par la suite, les rapports internes font état d'un tirage annuel de 10000 exemplaires. Ces résultats assurent la rentabilité du service du budget familial.

Néanmoins, pour les dirigeants nationaux, la vente intensive du cahier est un objectif louable mais insuffisant. Il faut aussi faire l'éducation budgétaire pour provoquer chez ses utilisateurs le besoin de prendre en charge leur condition de consommateur. Ce projet a eu quelques suites. Ainsi la LOCF collabore volontiers avec l'Association canadienne des consommateurs fondée en 1948. Plusieurs militants des deux branches de la Ligue se sont retrouvés dans les coopératives de consommation (des épiceries généralement) qu'ils ont souvent contribué à fonder.

C'est indéniablement dans le secteur du logement que l'implication sociale de la LOC a été la plus constante et a connu le plus grand succès ${ }^{36}$. À nouveau, les questions morales se mêlent aux considérations pratiques. En effet, dès 1940, l'intérêt des locistes pour la question de l'habitation suit, logiquement, les perspectives ouvertes par le catholicisme social qui, dans sa quête d'une troisième voie entre le capitalisme et le socialisme, définit la propriété comme un droit individuel et social. D'ailleurs, la LOC se contente, dans un premier

34. La LOC reprend ici le modèle, courant avant 1960, du partage des rôles dans la gestion des loisirs au Québec: la municipalité se borne à fournir les espaces aménagés, les équipements et les ressources financières; les associations volontaires, telles les Euvres des terrains de jeux, gardent la maîtrise d'œuvre des activités. Voir Michel Bellefleur, L'Église et le loisir au Québec, avant la Révolution tranquille (Québec, Les Presses de l'Université du Québec, 1986).

35. Les droits d'auteur de la LOC sont alors vendus aux Éditions de l'Axe (service de la Librairie du Centre catholique Inc.) de Drummondville.

36. Pour une analyse détaillée de l'implication de la LOC dans la question du logement entre 1939 et 1954, voir Jean-Pierre Collin, op. cit., chapitres 6 et 7 . 
temps, d'un réformisme moraliste qui n'apporte rien de nouveau aux positions défendues depuis trente ans, au moins, par les organisations catholiques $^{37}$.

Toutefois, accordant progressivement à la solution de ce problème un rôle stratégique dans la régénération économique et sociale du monde ouvrier, son intérêt pour la question devient plus marqué. Les locistes contribuent à élaborer un programme de revendication, le crédit urbain, qui traduit surtout un thème cher au catholicisme social officiel au début des années 1940: la diffusion de la propriété résidentielle parcellaire parmi les ouvriers. À ce stade, la question du logement et celle de la propriété ouvrière se confondent.

Mais graduellement, au fil des actions qui s'inscrivent dans sa «campagne de l'habitation» amorcée à l'hiver 1944-1945, on observe un net élargissement des perspectives de la Ligue, autant au plan du diagnostic que dans la recherche de solutions.

Cette évolution vers une implication davantage sociale qu'apostolique se condense, finalement, dans la participation massive des locistes à l'organisation du mouvement coopératif d'habitation. Au détriment parfois de leurs obligations envers la LOC elle-même, ils en guident le développement. Localement, ils s'impliquent, selon diverses formules, dans l'animation et l'administration des coopératives. D'une vingtaine en 1948, le nombre des coopératives québécoises d'habitation passe à 95 en 1951. Dans plus de la moitié des cas, la mise en place de ces coopératives fait suite aux cercles d'études organisés et animés par les locistes ${ }^{38}$.

Sur le plan national, la LOC exerce un contrôle très étendu sur le principal regroupement provincial. Tout en bâtissant des entreprises capables de loger des salariés en les rendant propriétaires, la Ligue cherche à faire des coopératives des agents de changement social, et des coopérateurs des citoyens responsables.

Car l'objectif principal de la Ligue demeure de faire en sorte que la production du logement et la gestion du parc résidentiel relèvent d'une action communautaire soutenue et durable. Pour sa part, elle réussit à faire prévaloir, entre 1948 et 1952, un véritable projet coopératif. Dans ce projet, dépassant la lutte contre la prolétarisation, la coopérative d'habitation sert de point d'appui à l'expérimentation de

37. Les principaux éléments de ce discours ont été exposés et analysés dans Marc $\mathbf{H}$. Choko, Crises du logement à Montréal (1860-1939) (Montréal, Éditions Albert Saint-Martin, 1980).

38. Certaines sections locistes masculines se dotent d'une coopérative d'habitation et d'autres se transforment même en coopératives d'habitation. 
villages coopératifs, des environnements résidentiels neufs dotés d'un ensemble intégré d'institutions communautaires.

Ce modèle a connu un succès intéressant dans plusieurs agglomérations urbaines ${ }^{39}$, à Sherbrooke, dans la Mauricie et dans les BoisFrancs de même que, plus tardivement, dans l'agglomération montréalaise $^{40}$.

\section{UNE DÉCONFESSIONNALISATION EN DOUCEUR}

Exception faite du secteur de l'habitation, les «grands services» affichent des performances inférieures aux attentes et de courte durée. Malgré tout, leur développement introduit une dissociation partielle mais soutenue avec l'institution ecclésiale. Les militants les plus impliqués sont «sortis» de la paroisse. Ils se retrouvent, en effet, dans des cercles d'étude fédéraux ou au cercle d'étude national. Le militantisme dans les services s'ajoute à celui qui doit avoir lieu dans les sections locales et déplace le centre d'intérêt de ceux qui s'y engagent à fond. Cela a d'autant plus d'impact que, la multiplication des forums sur le crédit urbain et le budget familial en ayant accéléré le rythme, des dirigeants nationaux projettent, pendant quelque temps, de relier le recrutement des membres aux grands services de l'Entr'aide familiale ouvrière.

Au printemps de 1946, en effet, le comité national fait imprimer 50000 exemplaires d'un livret dont le contenu a été discuté en conseil national: Un coup de main, les amis. Désireux d'ouvrir la LOC à toutes les familles ouvrières, on les invite à joindre ses rangs pour construire le réseau des services. C'est dans ce contexte que l'on envisage d'adopter un nouveau nom - «Mouvement d'Entr'aide Familiale» -, voire de changer les structures de l'organisation, en intégrant des milliers de membres.

S'exprime alors, par diverses propositions et tentatives de réformes, un courant implicitement favorable à une déconfessionnalisation en douceur de l'Action catholique ouvrière adulte, qui n'est pas sans rappeler la transformation de la LOC française en Mouvement populaire des familles (MPF) ${ }^{41}$. Déjà, sans renier ses attaches religieuses et sa fidélité à la doctrine sociale de l'Église, Le Front ouvrier, dans ses structures et dans sa présentation, n'insiste pas sur ses origines catholiques. Cela lui vaut, d'ailleurs, d'être l'objet de la

39. Jean-Pierre Collin, op. cit., 290-304.

40. Jean-Pierre Collin, op. cit., et «Crise du logement et action catholique à Montréal, 1940-1960», Revue d'histoire de l'Amérique française, 41,2 (automne 1987): 179-203.

41. Là-dessus, voir Joseph Debes, Naissance de l'Action catholique ouvrière, op. cit. 
critique acerbe de la part des milieux catholiques plus conservateurs ${ }^{42}$. Les initiatives de la Ligue en matière de services sociaux évitent de se présenter sous enveloppe catholique. Mieux, comme le montre son implication dans les coopératives d'habitation, le mouvement manifeste une prédilection pour les solutions coopératives sans attaches confessionnelles officielles.

L'expérience de la LOC nous rappelle une nouvelle fois qu'au Québec, dans plusieurs domaines, la déconfessionnalisation des institutions socio-économiques s'est faite de manière progressive ${ }^{43}$ et en grande partie de l'intérieur même de l'Église. Raymond Courcy a insisté sur cet aspect dans son étude du rôle social et politique de l'Église dans les quartiers populaires de Québec et de Montréal au cours de la deuxième moitié des années 1970. Il rejoint ainsi les analyses qui ont été faites de la CTCC-CSN ${ }^{44}$, du cas des loisirs en milieu urbain ${ }^{45}$, de l'évolution des pratiques dans le champ du travail social ${ }^{46}$, voire de la révolution administrative amorcée dans les années $1950^{47}$.

En outre, les propositions et tentatives de réformes de la LOC formulées au sortir de la guerre sont aussi un indice que l'importance de l'Entr'aide découle moins des champs spécifiques d'intervention retenus que de la perspective globale qu'elle veut introduire.

42. La critique porte en particulier sur le nom du journal dont on dénonce, à plusieurs reprises, l'allure communiste. En 1951 encore, le président du conseil d'administration juge bon «(d'attirer) l'attention des membres du Conseil sur certaines remarques faites tant par les vendeurs d'abonnements et de publicité que par certains curés au sujet du nom du journal qui le fait croire 'communiste' et (de mentionner) que ces observations seront étudiées». (Rapport du Conseil national de la LOC des $17-18$ novembre 1951, p. 8 ).

43. «Au cours des années 1950-1960, observe avec à-propos Fernand Dumont, nous n'avons pas assisté à de violentes contestations. L'Église s'est désistée, sans grande polémique, sans de fermes résistances, de son emprise sur l'éducation, les services sociaux, les organisations syndicales et autres» (Fernand Dumont, «Mutations de la culture religieuse dans le Québec francophone», Canadian Issues/Thèmes canadiens, 7 (1985): 15 et 16).

44. Jacques Rouillard, «Mutations de la Confédération des travailleurs catholiques du Canada, 1940-1960», Revue d'histoire de l'Amérique française, 34,3 (décembre 1980) et LouisMarie Tremblay, Le syndicalisme québécois: idéologies de la C.S.N. et de la F.T.Q., 1940-1970 (Montréal, Les Presses de l'Université de Montréal, 1972).

45. Michel Bellefleur, op. cit.

46. Hélène Pelletier-Baillargeon, Marie Gérin-Lajoie (Montréal, Boréal Express, 1985); Marta Danylewycz, Taking the Veil: An Alternative to Marriage, Motherhood, and Spinsterhood in Quebec, 1840-1920 (Toronto, McClelland and Stewart, 1987); Lionel Groulx et Charlotte Poirier, «Histoire de l'École de service social de l'Université de Montréal (1939-1976)», Intervention, 69 (juillet 1984). Jacques Palard, «Le 'travail social' au Québec: de la logique religieuse à la rationalité étatique», Service social, 31,1 (1982).

47. Hubert Guindon, «Réexamen de l'évolution sociale du Québec», Marcel Rioux et Yves Martin, dir., La société canadienne-française (Montréal, Hurtubise HMH, 1971), 166-168. 


\section{PROPAGER L'HABITUDE}

\section{DE L'ORGANISATION COMMUNAUTAIRE}

«Le problème (ouvrier) ne se résume pas à un problème matériel, mais c'est cela qu'il est avant tout ${ }^{48} \gg$. Partant d'observations de ce genre, l'Entr'aide familiale ouvrière résulte, plus particulièrement, de la prise de conscience que l'ouvrier vit ce problème non seulement comme travailleur et salarié, mais aussi comme consommateur des biens de première nécessité et comme usager des services publics. C'est pour répondre à cette autre dimension de la détresse physique (matérielle) et morale de la classe ouvrière que les services en général, et l'EFO en particulier, ont été organisés.

Bien entendu, certains aspects des conditions de vie hors travail peuvent être réglés sur le lieu du travail. Dans cet esprit, par exemple, les rédacteurs du Front ouvrier se montrent relativement sympathiques aux comités paritaires d'entreprises, une formule d'inspiration corporatiste importée de France par l'Association professionnelle des industriels (API). Mais ces solutions, considère-t-on, seront toujours partielles eu égard à la complexité, à la variété et à l'évolution constante des problèmes sociaux qui confrontent le monde ouvrier. Les réponses durables, estime-t-on, sont celles qui s'adressent plutôt à l'usager ou au bénéficiaire au lieu même de la consommation de biens et de services, c'est-à-dire, la famille et, à la rigueur, la paroisse ou le quartier.

Dans ce contexte, l'animation et la gestion de services servent d'abord à répondre à des besoins spécifiques de la vie quotidienne identifiés par les militants, à l'occasion notamment de l'enquête sociale et de la semaine de la famille ouvrière. Par conséquent, ils doivent «être ouvert(s) sur la masse» et non pas réservés seulement aux locistes.

Cet objectif primaire ne peut toutefois justifier à lui seul que la LOC prenne en charge des problèmes sociaux. À moyen terme, ajoute-t-on, «les services de la L.O.C. (devront être) ses chars d'assaut pour la conquête et la transformation du milieu social ${ }^{49}$ ». «Servir pour conquérir». Dans son sens restreint, ce slogan veut dire que les services sont un moyen particulièrement efficace pour soutenir l'expansion de la LOC, un moyen pratique de dépistage et de recrutement de nouveaux militants et, plus largement, un moyen d'identification de l'élite ouvrière. Ainsi, les jardins ouvriers sont-ils proposés, année

48. «Aura-t-elle des suites?», Le Front ouvrier, 1,8 (20 janvier 1945): 4.

49. Meneurs (bulletin mensuel interne de la LOC), 8,2 (novembre 1947): 3. 
après année, comme un moyen de recruter des «chefs» en même temps que des jardiniers.

Cependant, les militants doivent prendre garde de se faire piéger par les services, de verser dans «l'agitation catholique», perdre de vue l'essentiel et oublier la LOC proprement dite. C'est pourquoi on insiste tout particulièrement sur le second sens du slogan «Servir pour conquérir»: générer et entretenir un terrain favorable à l'éducation populaire.

Ainsi, les services fournissent au lociste l'occasion de prêcher par l'exemple. Quand il organise pour lui-même et ses proches des loisirs sains, en famille, quand il gère avec intelligence son patrimoine et son revenu familial, quand il se révèle un coopérateur exemplaire, etc., le lociste fait la preuve que la famille ouvrière peut prendre en main ses problèmes et leur apporter des solutions originales. Cette œuvre d'éducation sociale doit, premièrement, servir à convaincre la famille ouvrière de l'urgence de remplir ses devoirs avant de réclamer ses droits. De la même manière que pour poser les jalons de la collaboration entre le travail et le capital et pour prétendre au droit de participation dans l'entreprise, le travailleur doit faire preuve à l'usine ou au travail de «conscience professionnelle», la famille ouvrière, argumente-t-on, doit «remonter le courant (et) prendre ses responsabilités [...] même si les autres ne [...] suivent pas ${ }^{50}$ ».

Ce discours moralisateur, qui fait porter aux familles ouvrières, et plus spécifiquement aux femmes ouvrières, le fardeau de la preuve qu'elles méritent d'être prises en considération et aidées, est un des leitmotiv du journal. Numéro après numéro, on exige de la famille ouvrière qu'elle fasse preuve de modération en tout, qu'elle gère bien ce qu'elle a, qu'elle évite les pièges de plus en plus nombreux que lui tend la vie moderne, etc. Bref, la famille ouvrière doit apprendre à faire passer les dures réalités de la vie avant les plaisirs faciles; elle doit savoir discerner entre ses besoins réels et ceux qu'elle se crée et, pour améliorer son sort, orienter en conséquence ses ressources et ses énergies.

À l'origine, le choix des secteurs d'intervention et l'organisation des grands services est toujours fait tant pour des motifs moraux que pour des considérations économiques. Mais en passant du discours aux réalisations pratiques, l'Entr'aide familiale ouvrière ajoute à cet enseignement moraliste et à cette entreprise de «contrôle social», que l'on retrouve aussi bien dans l'ensemble des œuvres catholiques, un autre message: la famille ouvrière ne doit pas attendre que les solu-

50. «Famille ouvrière, tu y regagnes!», Le Front ouvrier, 6,24 (13 mai 1950): 11. 
tions viennent des spécialistes ou des pouvoirs publics, mais d'ellemême et de ses semblables. Les familles ouvrières, comme consommatrices et bénéficiaires, ont des problèmes spécifiques qu'elles sont seules à pouvoir définir. En plus d'apprendre aux familles ouvrières à effectuer dans l'immédiat les bons choix, faire l'éducation populaire par les services signifie créer des occasions qui feront comprendre aux ouvriers que «la réponse à leurs problèmes, ils la trouveront probablement d'eux-mêmes, dans l'union ${ }^{51} \gg$. La famille ouvrière doit faire taire son individualisme et acquérir le réflexe de l'organisation ${ }^{52}$.

L'organisation de l'Entr'aide familiale ouvrière a donc, avant tout, une visée pédagogique. Elle est une école de formation à la coopération $^{53}$. Cette entreprise de coordination et d'organisation d'un réseau polyvalent de services sociaux dans tous les domaines de la vie quotidienne (économie familiale, habitation, loisir, santé, etc.) doit être conçue comme un instrument d'un projet d'ensemble. Son importance tient moins aux champs spécifiques d'intervention retenus et aux performances quantitatives de ses services qu'à la perspective «communautaire» qu'elle veut introduire.

Bref, au syndicalisme déjà en marche, il faut ajouter une deuxième force, celle de la coopération, non pas dans le sens restreint de multiplier les institutions coopératives incorporées sous la loi des syndicats coopératifs - encore qu'il faille encourager les militants à le faire chaque fois que des aspects économiques (ou financiers) sont en jeu - mais dans celui de faire surgir une «mystique de la coopération», en vue de renouveler les «cadres sociaux» du milieu ouvrier. Une formule, copieusement reprise dans le journal et dans les bulletins internes, résume le sens de ce programme d'action: «des coopérateurs avant la coopérative».

Évidemment, ceux que l'on touche par les services ne deviennent pas tous des militants locistes. On espère toutefois en faire des «coopérateurs» qui auront, chaque fois qu'un problème collectif se pose, dans une variété de formes et de formules, le réflexe de l'organisation communautaire. Dans cette perspective, la LOC fait non seulement appel au principe de la subsidiarité ${ }^{54}$, elle tente également

51. Pour la conquête des foyers ouvriers (bulletin mensuel interne de la LOC), 12,7 (mars 1946): 6.

52. Réginald Boisvert, «On nous souhaite bonne fête», Le Front ouvrier, 4,41 (4 septembre 19.48): 4.

53 LOC de Montréal, Le catéchisme de la L.O.C. (Montréal, 1945), 26.

54. L'autorité politique doit éviter de «retirer aux groupements d'ordre inférieur, pour les confier à une collectivité plus vaste et d'un rang plus élevé, les fonctions qu'ils sont en mesure de remplir eux-mêmes. L'objet de toute intervention en matière sociale est d'aider les membres du corps social, et non pas de les détruire ni de les absorben» (Pie XI, Quadragesimo anno 
d'en renouveler la forme et le contenu par la création d'un réseau multiforme, et pas obligatoirement confessionnel, d'entreprises communautaires, de coopératives et d'associations diverses.

\section{LA RESPONSABILITÉ SOCIALE DE L'ÉTAT}

Bien sûr, il ne s'agit pas de décharger la collectivité ou les gouvernements de leurs responsabilités sociales. Au contraire, on rappelle volontiers que, pour faire la preuve que des réformes sont possibles et souhaitables, que la construction d'un monde plus humain est possible, que la nature des changements doit être déterminée par les premiers concernés, il faut que les pouvoirs publics participent activement à la recherche d'un nouvel ordre économique. Dans cette «Cité chrétienne», sera corrigée l'exploitation de l'ouvrier comme travailleur et salarié mais aussi comme consommateur. Tout en acceptant l'essentiel des règles de l'économie de marché, fondée sur le respect de l'initiative et de la propriété privées, les gouvernements ont l'obligation d'intervenir par une politique sociale qui déplace la finalité du régime économique des impératifs de la production (autrement dit des profits) vers les besoins de la personne ${ }^{55}$.

Pour mener à bien cette entreprise d'humanisation du régime économique, l'État n'a pas besoin de prendre à sa charge ou de normaliser la production directe de biens ou de services chaque fois qu'un problème se pose, comme le suggèrent, par exemple, le rapport Marsh et tous ceux qui s'en inspirent. Il convient beaucoup mieux, estime-t-on, d'assurer aux ouvriers «leur minimum vital» par un ensemble de mesures et de stratégies dont l'effet global sera de garantir le «juste salaire» à la famille ouvrière. Inlassablement, la LOC revient à la charge pour réclamer de l'État ce salaire familial ${ }^{56}$ dont elle fait «le fondement de toute autre revendication ou de toute

(Montréal, Institut social populaire, 1968), 33-34). Selon ce principe de la subsidiarité (voir aussi dans Jean XXIII, Mater et magistra (15 mai 1961) (Paris, Spes, 1965), les paragraphes 53 et 65), l'État doit céder la place aux communautés naturelles chaque fois que leur action est pertinente et efficace, et respecter pleinement leur autonomie par rapport au politique (Clinton Archibald, Un Québec corporatiste? (Hull, Éditions Asticou, 1983), 113-144) et leur diversité.

55. Livrée par bribes par tous les rédacteurs du Front ouvrier, cette vision du nouvel ordre économique et du rôle social de l'État - pour laquelle on utilise à plusieurs reprises une expression empruntée à Jacques Maritain, «l'économie distributive» - est développée avec détails et nuances par Fernand Bourret, dans ses éditoriaux et dans plusieurs articles d'analyse parus dans Le Front ouvrier, entre juin 1949 et juin 1950. Il a publié, à la même époque, un essai sur le même sujet: Scandale de l'économie (Introduction à l'économie de demain) (Montréal, Les Éditions ouvrières, 1950), 238 pages.

56. Pratiquement, le salaire familial est généralement défini comme le niveau de revenu permettant de répondre à tous les besoins essentiels d'une famille ouvrière de taille moyenne, c'est-à-dire, dans le cas du Québec, une famille de cinq personnes. Voir, par exemple, Le Mouvement ouvrier, 4,12 (septembre 1943): 8-9 et Le Front ouvrier, 4,12 (14 février 1948): 4. 
autre amélioration dans la situation ouvrière ${ }^{57}$ 》. Par son insistance $e^{58}$ à défendre, au nom de la justice distributive, la notion de salaire familial et les politiques privées et publiques qui s'y rattachent, la LOC se rapproche, nettement, du catholicisme «progressiste» américain ${ }^{59}$.

En touchant un tel salaire familial, la famille ouvrière pourra s'organiser des services sociaux coopératifs, s'offrir des loisirs, faire ses propres choix et sortir de sa situation prolétarienne. Bref, elle pourra prendre en charge ses problèmes de logement, de santé, d'éducation, de loisir, etc. D'où la nécessité de concevoir le syndicat (union, corporation professionnelle, etc.), la coopérative et les autres institutions du milieu ouvrier non seulement comme des coalitions momentanées en vue d'obtenir un gain immédiat et personnel, mais comme des institutions aussi permanentes qu'un gouvernement, capables à la longue de faire prévaloir une manière d'organiser les rapports économiques dans une économie de marché. Évidemment, la forme et le mandat spécifiques des organisations pourront varier au gré de la conjoncture. Ce qui doit demeurer, ce qui doit être constant, c'est la perspective coopérative ou communautaire avec laquelle sont abordés les problèmes sociaux.

57. La rédaction, «Organisons-nous», Le Mouvement ouvrier, 5,9 (juin 1944): 4.

58. La multiplicité des efforts consacrés à l'organisation du service du budget familial pendant près de dix ans et l'empressement avec lequel on en fait un service national témoignent de l'importance accordée à cette revendication. La pratique du budget familial doit servir à mieux gérer les finances de la famille et, partant, à discipliner ses choix et ses comportements. Mais la LOC veut aussi en faire une arme politique. Bien qu'incapables, devant l'envergure de la tâche, de donner suite à ce projet, les dirigeants nationaux soutiennent, à plusieurs reprises, que la compilation de dizaines de milliers de budgets tenus soigneusement depuis un an ou plus serait une démonstration convaincante à l'appui de la nécessité de légiférer pour redresser le salaire familial. Le mouvement doit être capable de produire ses propres statistiques qu'il pourra confronter à celles du gouvernement et forcer ainsi l'adoption de mesures qui assureront à la famille ouvrière un revenu minimum garanti.

59. Celui-ci, en effet, dans un effort d'établir sa propre tradition du «social gospel», en a fait l'élément central de son programme de reconstruction sociale depuis l'adresse collective des évêques des États-Unis au sortir de la Première Guerre mondiale («The Bishop's Program of Social Reconstruction», reproduit dans Aaron I. Abell, ed., American Catholic Thought on Social Questions (New York, The Bobbs-Merrill Company, 1968), 325-347). Cette revendication du salaire (ou revenu) familial est principalement inspirée des travaux du théologien et économiste John A. Ryan qui, en s'appuyant sur Rerum novarum et plus tard sur Quadragesimo Anno, insiste tout particulièrement sur la responsabilité de l'État en cette matière - une attitude qui est loin d'être partagée par tous les interprètes du catholicisme social. Voir Charles E. Curran, American Catholic Social Ethics: Twentieth-Century Approaches (Notre Dame, Indiana, University of Notre Dame Press, 1982), 30 et s. et Jay P. Dolan, The American Catholic Experience: a History from Colonial Times to the Present (New York, Doubleday, 1985), 342-346 et 401417. 


\section{UN MODÈLE SOCIAL DE GESTION DES BESOINS COLLECTIFS}

Ainsi, dans les années 1940, profitant de son implantation dans le milieu urbain québécois, la LOC suggère et expérimente un modèle social (non étatique) de gestion des besoins collectifs. Tout en n'étant pas exempte de conservatisme et de moralisme, cette amorce d'un syndicalisme du cadre de vie n'équivaut pas strictement à défendre les institutions (catholiques) de service social déjà en place, d'autant plus que, même si ses dirigeants n'en font pas un objet de controverse comme ce fut le cas au sein du mouvement coopératif, il nous a semblé exister, à l'intérieur même de la LOC, un courant implicitement favorable à la déconfessionnalisation sans éclats de l'Action catholique ouvrière. Ainsi, la Ligue propose, dans un esprit d'affirmation et d'actualisation du principe de subsidiarité, un des points d'appui du catholicisme social, de créer de nouvelles institutions de service social adaptées à ce qu'elle considère être les caractéristiques de la classe ouvrière et de la vie urbaine.

Par ailleurs, loin d'appuyer une politique du laisser-faire, l'application du principe de la subsidiarité s'accompagne d'une ouverture à une certaine forme de politique sociale, voire d'un appel à l'intervention des pouvoirs publics. Toutefois, par l'accent qu'elle met sur la gestion sociale des problèmes sociaux, la LOC véhicule un autre choix que celui de l'État providence: par conservatisme peut-être — ou probablement - , mais également parce qu'elle a de la totalité sociale une perception différente, qu'elle tire de son adhésion au catholicisme social.

L'attitude critique, voire l'opposition farouche de la LOC à l'endroit de l'intervention de l'État dans le champ des politiques sociales, est plus qu'un pur réflexe de défense d'un ordre établi. Malgré certaines professions de foi, ce choix n'est pas orienté principalement vers la réforme des structures et vers l'implantation d'un programme général de restauration sociale qui serait le prélude à l'établissement d'une «troisième voie», à côté du capitalisme et du socialisme. Pour reprendre le schéma d'analyse proposé par Louis Maheu, à propos des nouveaux mouvements sociaux du tournant des années 1980, l'important pour la LOC n'est pas de bâtir un nouveau cadre politique qui rendrait possible une «appropriation étatique du social». Elle veut, au contraire, faire prévaloir une «appropriation collective du tissus social ${ }^{60}$ ».

60. Louis Maheu, «Les mouvements de base et la lutte contre l'appropriation étatique du tissu social», Sociologie et sociétés, 15,1 (1983): 77-92; «Crise sociale, mouvements sociaux et pratiques du changement social», Politique, 4 (automne 1983): 27-54. 
Bref, dans les années 1940 tout au moins, la LOC souhaite voir surgir, au sein de la société civile, un nouveau maillage institutionnel grâce auquel la famille ouvrière pourra s'organiser des services sociaux coopératifs, faire ses propres choix et sortir de sa situation prolétarienne.

La Ligue participe ainsi, à sa manière, au renouvellement de la pensée catholique au Québec. Son action s'ajoute à celle des partisans d'un nouvel humanisme chrétien ${ }^{61}$ qui, durant les années 1940 , travaillent à implanter un certain pluralisme idéologique dans l'Église. L'histoire de la LOC montre, en particulier, que la diversification des points de vue dans l'Église déborde les cercles d'intellectuels. Son approche des problèmes sociaux, tout en étant fortement imprégnée de conservatisme et de moralisme, a vraisemblablement contribué à revitaliser le projet catholique d'action sociale, en le faisant sortir de son prosélytisme et de sa fixation pour la direction des organisations sociales.

Ce projet sur le terrain de l'action sociale ${ }^{62}$ a certainement trouvé une source d'inspiration dans Vatican II et dans l'activité et le brassage d'idées qui l'ont entouré. Mais ses caractéristiques essentielles avaient été fixées, mises à l'essai même, dans les décennies précédentes. Les modes d'organisation et les méthodes d'intervention ${ }^{63}$ locistes constituent un héritage favorable à l'émergence, dans les années 1960, d'une animation sociale qui, bien que séculière et professionnelle, ne s'est jamais affranchie d'une certaine «déontologie catholique ${ }^{64} \gg$.

La LOC a été un des maillons du renouvellement, au sens large, des conceptions sociales et des comportements politiques au Québec et un élément précieux de sa sécularisation, tout en contribuant de

61. Les expressions sont nombreuses pour désigner ce courant. Pour l'ACS, Gabriel Clément parle d'«humanisme chrétien» (Histoire de l'Action catholique...) ou «d'humanisme contemporain» («L'Action catholique: les mouvements spécialisés à Montréal de 1930 à 1966»); Jacques Rouillard décrit la nouvelle idéologie de la CTCC comme de «l'humanisme démocratique» ou de «l'humanisme libéral»; les membres de l'API se voient comme des «humanistes pratiques» (voir Michèle Champagne, «Le mouvement patronal catholique et la démocratie industrielle. Étude comparée Belgique, France, Québec (1945-1960)», Ginette Kurgan-Van Hentenryk, dir., La question sociale en Belgique et au Canada XIX ${ }^{e}-X X^{e}$ siècles (Bruxelles, Les Presses de l'Université libre de Bruxelles, 1988), 217); Michael Behiels, enfin, fait référence à un «humanisme séculier» (secular humanism) (op. cit.), 92.

62. Cette interprétation a été défendue par Raymond Courcy dans sa thèse de doctorat sur la basse-ville de Québec et le quartier Pointe-Saint-Charles, à Montréal. Voir Le rôle social et politique de l'Église catholique...

63. Dans notre thèse, nous avons montré que cette observation vaut également pour les modèles de prise de décision locistes (chapitre 4).

64. L'expression est de Raymond Courcy. 
façon significative à la mise en place d'éléments de contestation et de critique de ce que plusieurs appellent la «modernisation politique».

En contrepartie, partagé entre la version orthodoxe du catholicisme social et sa version moderne (l'humanisme chrétien alors en vogue dans les milieux catholiques «progressistes»), le potentiel réformateur du mouvement aura été constamment amorti par son rattachement à l'Église institutionnelle. Reflétant les ambiguïtés inhérentes au catholicisme social et le mélange des positions, caractéristiques de la thèse de la troisième voie et du personnalisme, la LOC fut incapable de sortir de ses ambivalences. En conséquence, elle manqua de souffle et, au début des années 1950, vit la portée de son action sociale refrénée au profit de sa mission religieuse. En effet, l'histoire de la Ligue ne fut pas entièrement placée sous le signe du pragmatisme et du modèle communautaire de gestion des besoins collectifs. Au contraire. Au tournant des années 1950, et plus encore à partir de 1954 , le mouvement réévalue ses priorités et, dès lors, délaisse le champ de l'action sociale pour se replier, de façon générale, derrière sa mission apostolique. Le changement qui s'opère n'engage plus la Ligue sur le terrain de l'action sociale, mais sur celui de l'éthique sociale.

Parmi les facteurs internes qui expliquent ce virage apostolique, il faut d'abord souligner la perte de dynamisme causée par le départ, à tous les échelons, de plusieurs militants et de quelques militantes attirés par l'action coopérative et par l'action syndicale. La LOC aurait été, d'une certaine manière, victime de la réussite de son incursion dans le champ de l'action sociale. Mais l'évolution de la situation économique est aussi à mettre au nombre des causes.

Après la Seconde Guerre mondiale, le Québec entre dans une période de croissance économique presque continue qui se traduit, entre autres, par une augmentation graduelle du niveau de vie. Après quelques années, pour la majorité de la population des villes - dont une portion significative de la classe ouvrière -, les problèmes sociaux sur lesquels la LOC avait bâti son effort de mobilisation perdent temporairement de leur gravité. Mieux, ils semblent vouloir se résorber d'eux-mêmes. Devant une économie de marché qui paraît capable d'assurer aux ouvriers l'entrée dans la société de consommation, la perspective communautaire perd donc un peu sa raison d'être. Ce n'est qu'avec la récession de 1957 que l'on «redécouvre», à l'échelle de l'Amérique du nord, l'existence d'une pauvreté urbaine structurelle. On assiste alors à une recrudescence des initiatives visant à répondre aux problèmes toujours aigus de logement, d'alimentation, de santé, etc., notamment dans les zones d'urbanisation plus anciennes. 
Il faut rappeler aussi que l'apostolat social de la LOC n'a créé de commotion ni dans la société ni dans l'Église. Ce fut une entreprise relativement modeste dans ses réalisations, qui a duré quelques années à peine. La LOC, malgré ses prétentions répétées, n'a jamais entraîné la masse ouvrière à sa suite. Elle n'a pas non plus révolutionné la qualité de vie en ville.

Dans l'ensemble, l'influence directe de la LOC, aura été plus qualitative que quantitative. Ses réalisations ont été modestes en regard de ses ambitions initiales. La mise en œuvre de l'Entr'aide familiale ouvrière était forcément beaucoup plus lente que sa proclamation. L'organigramme de 1942 (figure 3) ne fut jamais qu'un cadre de référence, et la Ligue n'a pas tenu l'engagement, pris dans un élan d'optimisme lors du débat sur la réforme des structures du mouvement, de lancer un nouveau service chaque année. Néanmoins, l'Entr'aide familiale ouvière a ouvert des perspectives originales qui, à la fin de la décennie, forcent d'ailleurs les dirigeants nationaux à réfléchir sur les structures et sur l'avenir du mouvement.

\section{L'INTÉRÊT DES MOUVEMENTS SOCIAUX}

L'histoire de la LOC, notamment son implication dans la question du logement, permet, entre autres choses, d'illustrer un fait relevé par d'autres observateurs, à savoir que le double processus de modernisation politique et de sécularisation de l'Église et de la société québécoises ne se confond pas avec la technocratisation de la vie sociale associée à la professionnalisation de plusieurs domaines d'intervention ${ }^{65}$. Elle montre en effet qu'un mouvement social dont le projet de société était distinct de l'État providence a participé à l'avènement d'une société québécoise pluraliste, moderne et sécularisée.

Bien sûr, la LOC, comme un grand nombre de mouvements sociaux à la même époque, résiste à la conception «institutionnelle» du bien-être en remplacement de la conception «résiduelle ${ }^{66} »$. Surtout, dans leurs déclarations de principes, les locistes restent toujours d'ardents défenseurs des valeurs traditionnelles. Il faut toutefois éviter de les identifier seulement à ces déclarations et du même coup au

65. Hubert Guindon, Quebec Society: Tradition, Modernity, and Nationhood (Toronto, University of Toronto Press, 1988); Gilbert Renaud, «L'inversion tranquille. Le social québécois et ses métamorphoses», Yvon Desrosiers, dir., Religion et culture au Québec. Figures contemporaines du sacré (Montréal, Fides, 1986), 93-118; Jean-Jacques Simard, La longue marche des technocrates (Montréal, Éditions Albert Saint-Martin, 1979).

66. Sur cette évolution, voir Dennis Guest, The Emergence of Social Security in Canada (Vancouver, University of British Columbia Press, 1980); Yves Vaillancourt, op. cit. 
conservatisme. À leur manière, les locistes s'inscrivent dans le changement ${ }^{67}$.

Comme l'a montré une thèse récente, l'analyse du processus de redéfinition des politiques de bien-être social au Québec entre 1930 et 1960 s'accommode mieux du concept de "particularisme ${ }^{68}$ » que de ceux de décalage et de retard. D'ailleurs, plusieurs études ont démontré qu'en ce domaine, loin d'être fermée sur elle-même, la société québécoise était ouverte à une multiplicité d'influences. Ainsi, les efforts de redéfinition des pratiques en travail social ont-elles puisé à plusieurs sources: la sociologie de Le Play et le catholicisme social européen en général, le mouvement des Settlement Houses en GrandeBretagne, le service social américain, issu du Social.Gospel mais aussi d'un courant plus libéral au sein du catholicisme américain ${ }^{69}$.

Cependant, dans les années 1950, le modèle que les locistes proposent et expérimentent est en quelque sorte mis au rancart. Le libéralisme réformiste gagne alors l'adhésion de la classe politique montante. Chez les partisans comme chez les adversaires d'un nouveau partage des rôles et des pouvoirs entre l'État et l'Église, chez les tenants d'un nationalisme modernisé comme chez les apôtres de l'intégration à la société nord-américaine, la revalorisation du rôle de l'État à l'intérieur de l'économie de marché fait alors l'objet d'un large consensus. On tend à privilégier la scène politique comme lieu de résolution des conflits et comme mécanisme propre à faire progresser la société québécoise sur la voie de la modernité.

Dans ce contexte, le modèle communautaire, d'abord préoccupé de faire prévaloir une gestion sociale du social est vite rangé dans le camp du conservatisme et du nationalisme traditionaliste. Avec le recul, on est tenté de voir là un avortement du pluralisme qui se construit dans les années 1930 et 1940.

67. Ce diagnostic rejoint celui que posait récemment Yolande Cohen à propos des Cercles des fermières. Elle observe, par exemple, qu'à «leur manière, les Cercles nous font entrevoir comment les milliers de femmes qu'ils regroupent ont réussi à devenir des femmes modernes tout en restant des fermières» (Femmes de parole. L'histoire des Cercles de fermières du Québec, 1915-1990 (Montréal, Le Jour, éditeur, 1990), 263).

68. Peter Southam, Modernisation, question nationale et influences exogènes: le discours élitaire sur les politiques sociales au Québec, 1930-1960, thèse de doctorat (histoire), Université Laval, 1987. Dans le même esprit, Yves Vaillancourt (op. cit.) propose d'utiliser le concept de «différence».

69. Voir Hélène Pelletier-Baillargeon, Marie Gérin-Lajoie (Montréal, Boréal Express, 1985), 210-217 et 247-251; Jean-Pierre Deslauriers, «De l'animation à la révolution», Service social, $34,2-3$ (1985): 372. 